\title{
Transformation of Non-hypervascular Hepatobiliary Phase Hypointense Hepatic Nodules into Hypervascular Hepatocellular Carcinomas: regular MR follow-up results
}

Background: Non-hypervascular hepatic nodules showing hypointensity onhepatobiliary phase imaging (HBPI) in cirrhotic patientsare known to be premalignant.

Purpose: The risk oftransformation of non-hypervascular MR hepatobiliary phase imaging (HBPI) hypointense nodules into hypervascular hepatocellular carcinomas (HCCs) were evaluated.

Materials and Methods: Cirrhotic patients with non-hypervascular HBPI hypointense nodules were retrospectively analyzed if they were diagnosed as HCC and MR followed up longer than one year during the period starting from January 2010 to October 2016 with regular intervals of 3-6 months. Risk factors for the transformation into hypervascular HCCs were analyzed using the Cox proportional hazard model.

Results: Among the 103 nonhypervascular HBPI hypointense nodules meeting the inclusion criteria, transformation into hypervascular HCCs occurred in 44 tumors (42.7\%). Median follow-up period was 24 months. Multivariate analysis revealed that hyperintensity on T2weighted images (T2WI) or diffusion-weighted images (DWI) were the two independent predictors of transformation into hypervascular HCCs $(P=0.036$ and 0.041 , respectively). All 14 tumors with hyperintensity on $\mathrm{TWW}$ or DWI were transformed into hypervascular HCCs, and the 1- and 2-year transformation rates were $64.3 \%$ and $92.9 \%$, respectively. For the other 89 nodules without initial hyperintensity on T2W or DWI, the 1-, 2-, and 3-year transformation rates were $9.0 \%, 26.0 \%$, and $39.1 \%$, respectively. During follow-up, 21 of 29 (72.4\%) showing a new change on T2WI, DWI, or dynamic phases were transformed into hypervascular HCCs.

Conclusion: The transformation rates of HBPI hypointense nodules into hypervascular HCCs could be predicted according to the initial or serial MRI findings. It is not definite whether most HBPI hypointense nodules without initial hyperintensity on T2WI or DWI will be ultimately transformed into hypervascular HCCs.

Index Words: Liver Tumor, MR, Hepatocellular carcinoma, Malignant Transformation,

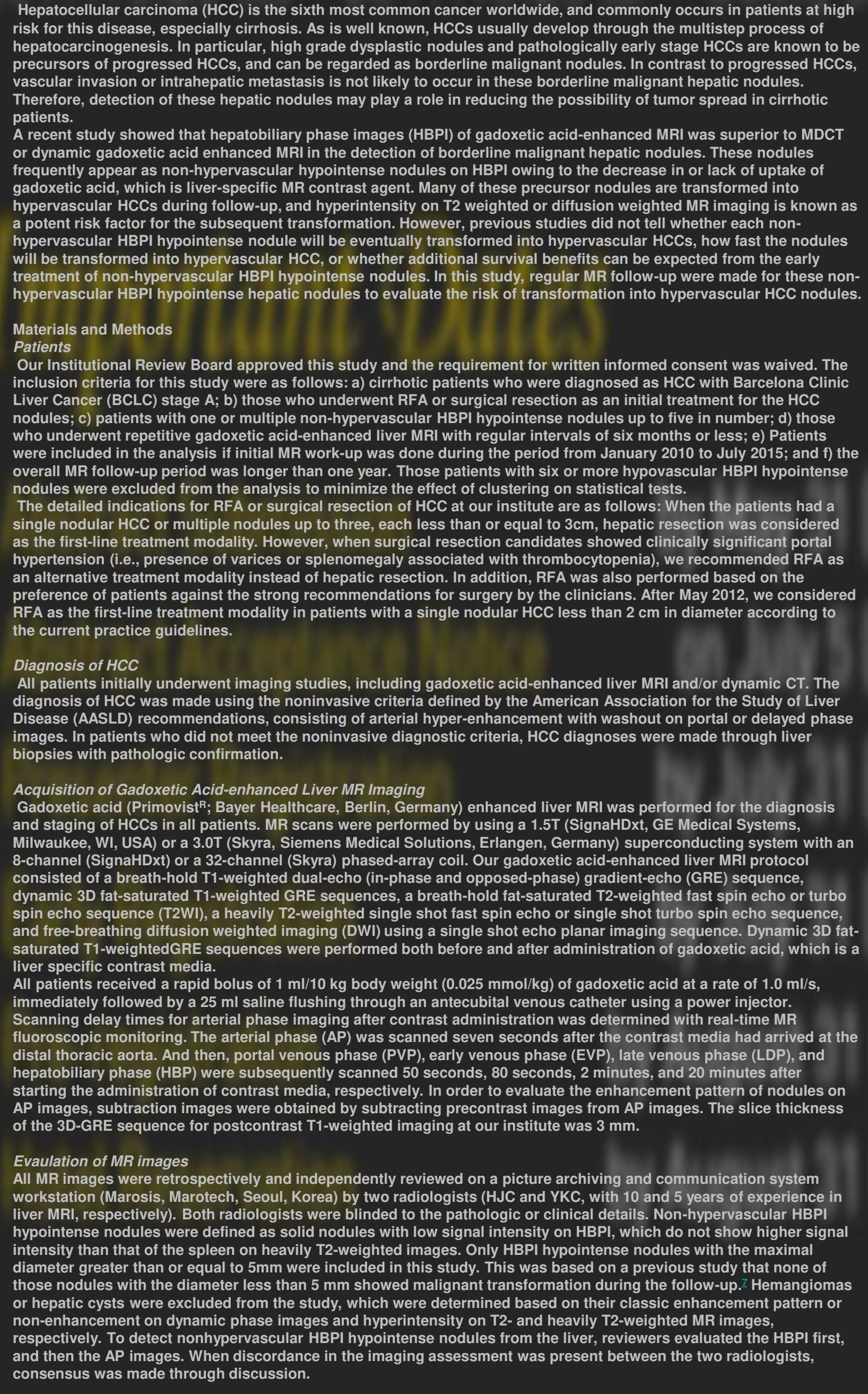

Statistical Analyst

proportional hazard model. Potential risk factors included patient's age and sex, tumor size, hyperintensity on T2WI or
DWI, multiplicity of HBPl hypointense nodules, baseline serum level of AFP, initital treatment modality for HCC, history of local therapy of hypervasuclar HCC nodules, Child-Pugh class, etiology of cirrhosis, and the existence of portal
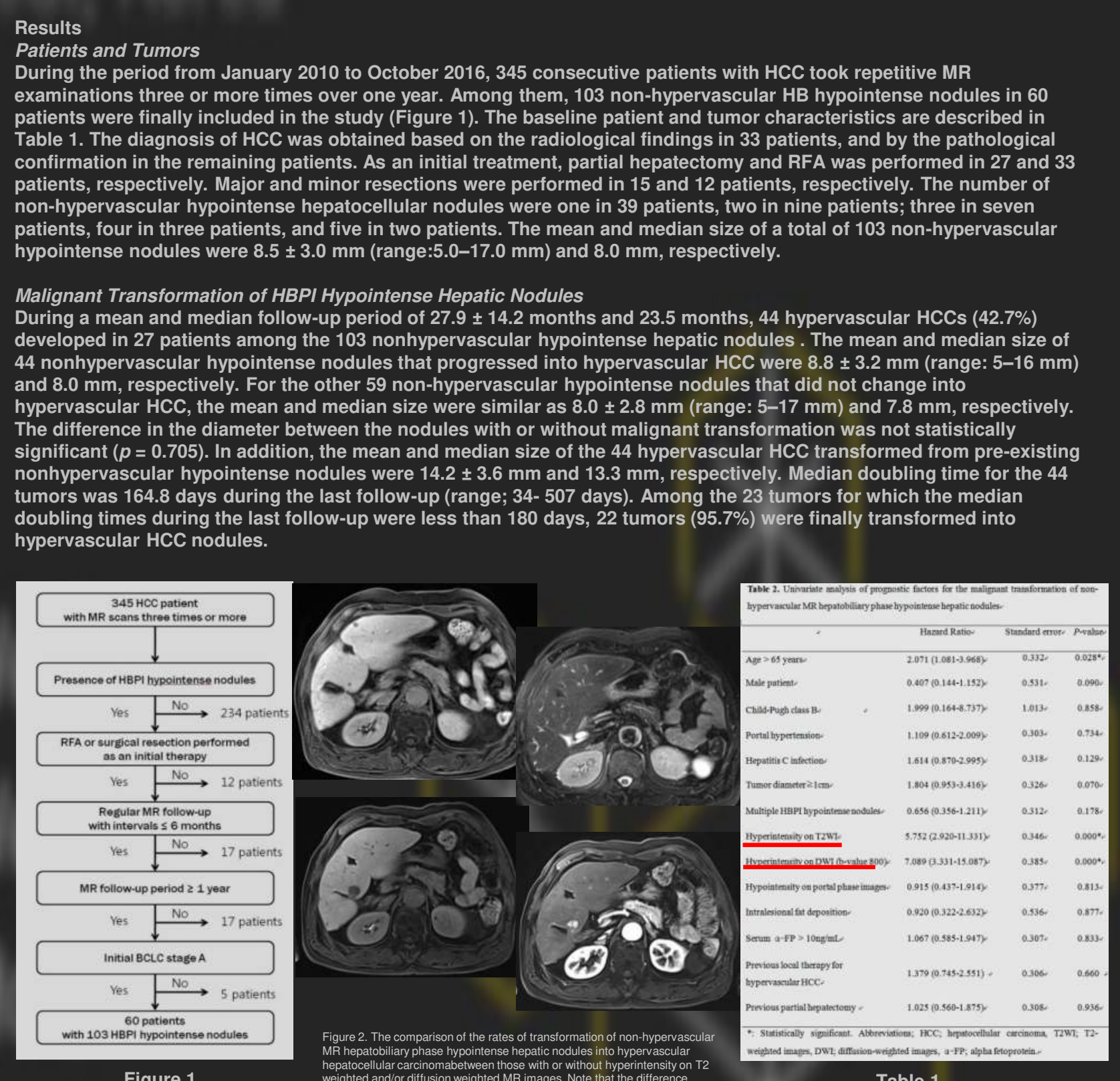

Figure
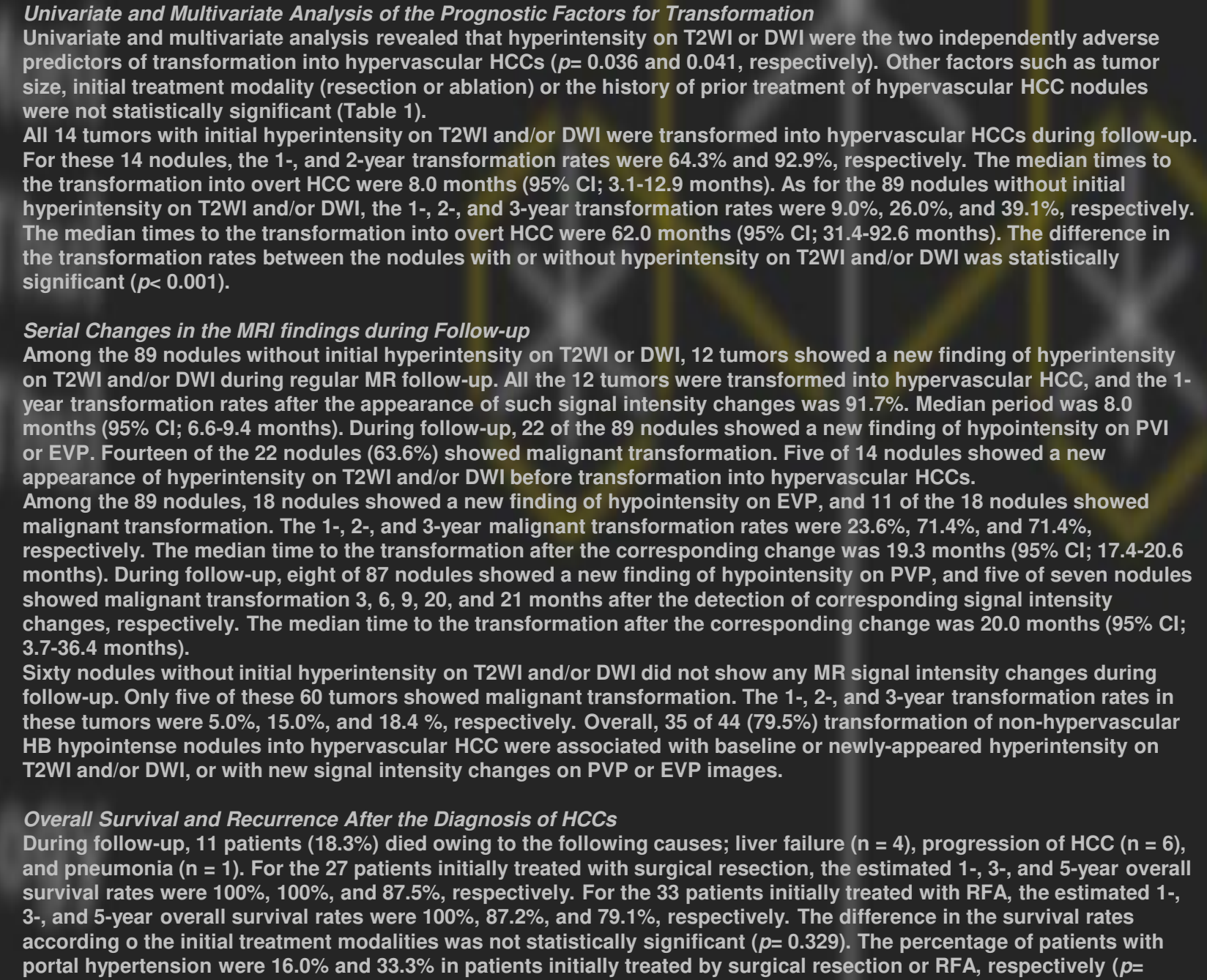

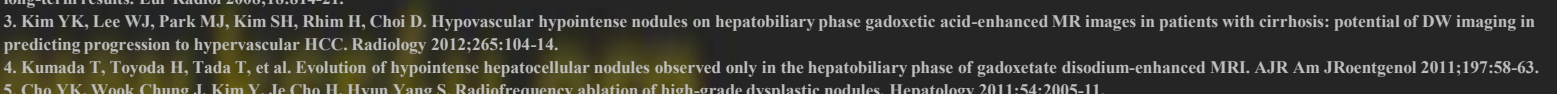

